\title{
Measurement of the Near Field Distribution of a Microwave Horn Using a Resonant Atomic Probe
}

\author{
Jingxu Bai ${ }^{1,2}$, Jiabei Fan ${ }^{1,2}$, Liping Hao ${ }^{1,2}$, Nicholas L. R. Spong ${ }^{3}$ and Yuechun Jiao ${ }^{1,2}$ \\ and Jianming Zhao ${ }^{1,2, * \text { (i) }}$ \\ 1 State Key Laboratory of Quantum Optics and Quantum Optics Devices, Institute of Laser Spectroscopy, \\ Shanxi University, Taiyuan 030006, China; jxbaisxu@163.com (J.B.); fan_jiabei@163.com (J.F.); \\ hao_lping@163.com (L.H.); ycjiao@sxu.edu.cn (Y.J.) \\ 2 Collaborative Innovation Center of Extreme Optics, Shanxi University, Taiyuan 030006, China \\ 3 Joint Quantum Centre (Durham-Newcastle), Department of Physics, Durham University, \\ Durham DH1 3LE, UK; nicholas.l.spong@durham.ac.uk \\ * Correspondence: zhaojm@sxu.edu.cn
}

Received: 8 October 2019; Accepted: 11 November 2019; Published: 14 November 2019

\begin{abstract}
We measure the near field distribution of a microwave horn with a resonant atomic probe. The microwave field emitted by a standard microwave horn is investigated utilizing Rydberg electromagnetically inducted transparency (EIT), an all-optical Rydberg detection, in a room temperature caesium vapor cell. The ground $6 \mathrm{~S}_{1 / 2}$, excited $6 \mathrm{P}_{3 / 2}$, and Rydberg $56 \mathrm{D}_{5 / 2}$ states constitute a three-level system, used as an atomic probe to detect microwave electric fields by analyzing microwave dressed Autler-Townes (AT) splitting. We present a measurement of the electric field distribution of the microwave horn operating at $3.99 \mathrm{GHz}$ in the near field, coupling the transition $56 \mathrm{D}_{5 / 2} \rightarrow 57 \mathrm{P}_{3 / 2}$. The microwave dressed AT spectrum reveals information on both the strength and polarization of the field emitted from the microwave horn simultaneously. The measurements are compared with field measurements obtained using a dipole metal probe, and with simulations of the electromagnetic simulated software (EMSS). The atomic probe measurement is in better agreement with the simulations than the metal probe. The deviation from the simulation of measurements taken with the atomic probe is smaller than the metal probe, improving by $1.6 \mathrm{~dB}$. The symmetry of the amplitude distribution of the measured field is studied by comparing the measurements taken on either side of the field maxima.
\end{abstract}

Keywords: microwave; near field; atomic probe; EIT-AT spectrum; stark effect

\section{Introduction}

Precise measurements of the strength, phase, polarity, etc. of electromagnetic (EM) fields are of great importance for science, communications, and biomedicine, having prominent roles in everyday life. Microwaves - electromagnetic waves with very high frequency (300 MHz to $3000 \mathrm{GHz}$ - play a very important role. In this study, microwaves are emitted from a horn. The radiation field of such a horn can be divided into three parts: the induction near field, the radiation far field, and an intermediate zone. Measurement of the induction near field is advantageous, as these measurements are convenient, have high fidelity, and can provide a large amount of characteristic information. The electric field distribution of a microwave horn can be used to understand the propagation of the microwaves as well as the electric field distribution in the far field, which can be difficult to measure. Traditionally, measurements of the microwave electric field are made using a dipole antenna as a probe. Probes are calibrated prior to measurement [1-3]. The accuracy is limited by the probe geometry, material, sensitivity, etc. With the development of atomic physics and detection technology, one can improve 
field measurement accuracy and sensitivity with an atom-based method [4-7]. Our atom-based field measurement has clear advantages over such traditional methods because it is based on invariant atomic properties and is therefore calibration free.

Rydberg atoms, with principal quantum number $n \gtrsim 10$, are an ideal candidate for measurement of the microwave field due to their huge dc polarisability $\left(\sim n^{7}\right)$ and microwave-transition dipole moments $\left(\sim n^{2}\right)$. This provides Rydberg atoms with extreme sensitivities to dc and ac electric fields [8]. At a high principle quantum number, the energy difference of Rydberg levels $\left(\sim n^{-3}\right)$ approaches the microwave-frequency range, leading to even stronger interaction between Rydberg atoms and microwave fields.

When the microwave field couples two close lying Rydberg states, the coupling strength is expressed with Rabi frequency $\Omega_{M W}=\mu_{M W} E / \hbar$, with microwave coupled dipole transition element $\mu_{M W}$, electric field $E$, and reduced Planck constant $\hbar$. The $\Omega_{M W}$ can be large when the electric field is weak because the $\mu_{M W}$ between neighboring Rydberg states scales as $n^{2}$. For the caesium transition $56 \mathrm{D}_{5 / 2} \rightarrow 57 \mathrm{P}_{3 / 2}$ used in this work, calculated $\mu_{M W}$ is $\sim 4258 e a_{0}$. Coupling between these two states due to the microwave electric field, $\Omega_{M W}$, causes the electromagnetically inducted transparency (EIT) peak to split into two, known as Autler-Townes (AT) splitting [9]. The magnitude of the AT splitting, $\gamma_{A T}$, is proportional to $\Omega_{M W}$, and so the magnitude of the splitting can be used to make an atom-based microwave field measurement. Rydberg EIT [10-12], a quantum coherence effect of the interaction between Rydberg atoms and lasers, will be employed to optically detect the microwave dressed AT splitting $\gamma_{A T}$ induced by the microwave field [13-29]. Additionally, we can use this kind of resonant Rydberg transition and fluorescence to detect $\mathrm{THz}$ radiation [30,31].

In this work, we first investigate the microwave near field distribution produced by a standard microwave horn employing microwave dressed Rydberg EIT-AT spectroscopy in a room temperature vapor cell. A $3.99-\mathrm{GHz}$ microwave field emitted by the horn couples the Rydberg transition, $56 D_{5 / 2} \rightarrow 57 P_{3 / 2}$, leading to AT splitting of the Rydberg EIT peak. The microwave EM near field distribution is extracted using measurements of the EIT-AT splitting as a function of the position of the atomic sensor in the near field of the horn. This measurement is compared to measurements taken with a traditional metal probe using a vector network analyzer (VNA). It is found that results from our Rydberg atom sensor show better agreement with electromagnetic simulation software (EMSS) simulation than measurements taken using a metal dipole probe.

\section{Experimental Setup}

Experiments were carried out in a caesium room-temperature vapor cell. A schematic of the experimental setup and relevant Rydberg EIT-AT four-level diagram is shown in Figure 1. The caesium ground state $\left|6 \mathrm{~S}_{1 / 2}, F=4\right\rangle$, excited state $\left|6 \mathrm{P}_{3 / 2}, F^{\prime}=5\right\rangle$, and Rydberg state $\left|56 \mathrm{D}_{5 / 2}\right\rangle$ are used to define a cascaded three-level EIT system, see Figure 1a. The coupling laser is resonant with the $\left|6 \mathrm{P}_{3 / 2}, F^{\prime}=5\right\rangle \rightarrow\left|56 \mathrm{D}_{5 / 2}\right\rangle$ Rydberg transition. A probe laser couples the lower $\left|6 \mathrm{~S}_{1 / 2}, F=4\right\rangle \rightarrow$ $\left|6 \mathrm{P}_{3 / 2}, F^{\prime}=5\right\rangle$ transition. The probe laser frequency is locked using polarization spectroscopy [32]. We scan the coupling laser across the transition, allowing us to obtain EIT spectroscopy without a Doppler background. A microwave electric field with a frequency $\sim 3.99 \mathrm{GHz}$ couples the $\left|56 \mathrm{D}_{5 / 2}\right\rangle \rightarrow$ $\left|57 \mathrm{P}_{3 / 2}\right\rangle$ Rydberg transition, forming microwave dressed EIT-AT spectroscopy.

Figure $1 \mathrm{~b}$ displays the experimental scheme. A weak probe beam, with a $1 / e^{2}$ waist of $100 \mu \mathrm{m}$ and a wavelength of $\lambda_{p}=852 \mathrm{~nm}$, and a strong coupling beam, with a $1 / e^{2}$ waist of $150 \mu \mathrm{m}$ and wavelength of $\lambda_{c}=510 \mathrm{~nm}$, are overlapped and in a counter propagating geometry through a vapor cell with diameter $20 \mathrm{~mm}$ and length $50 \mathrm{~mm}$. The probe and coupling beams are of the same linear polarization along $z$-axis. EIT microwave dressed AT splitting spectra are detected by measuring the transmission of the probe beam using a photodiode (PD) positioned after a dichroic mirror (DM). The power of probe and coupling lasers are $2.0 \mu \mathrm{W}$ and $29.8 \mathrm{~mW}$, respectively, which yield an EIT spectrum with a linewidth $2 \pi \times 5.08 \mathrm{MHz}$, as displayed in Figure 2a. The dominant peak at zero detuning corresponds to a double resonance, $\left|6 \mathrm{~S}_{1 / 2}, F=4\right\rangle \rightarrow\left|6 \mathrm{P}_{3 / 2}, F^{\prime}=5\right\rangle \rightarrow\left|56 \mathrm{D}_{5 / 2}\right\rangle$, while the 
small peak at $-168 \mathrm{MHz}$ results from the hyperfine structure of an intermediate state $\left|6 \mathrm{P}_{3 / 2}, F^{\prime}=4\right\rangle$ resonance, that is used to calibrate the coupling laser frequency. Here, the distance between two hyperfine EIT peaks, $168 \mathrm{MHz}$, is attained by considering Doppler mismatch factor, $\lambda_{p} / \lambda_{c}-1=0.67$, due to different wavelength of probe and coupling beams.

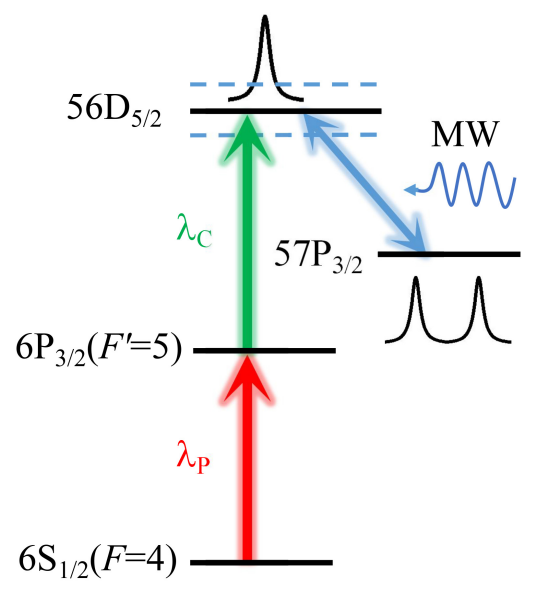

(a)

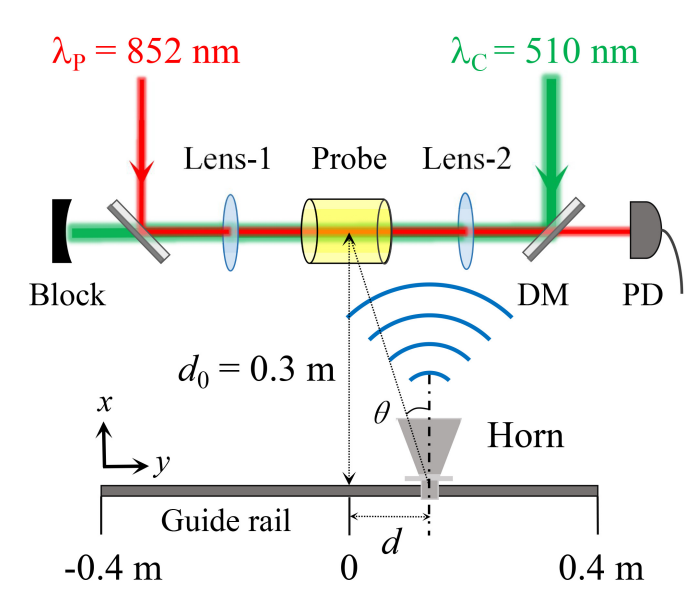

(b)

Figure 1. (Color online) (a) Energy level diagram of our caesium four-level system. A weak probe laser $\lambda_{p}$ is resonant with the lower transition $\left|6 \mathrm{~S}_{1 / 2}, F=4\right\rangle \rightarrow\left|6 \mathrm{P}_{3 / 2}, F^{\prime}=5\right\rangle$, and a strong coupling laser $\lambda_{c}$ scans over the Rydberg transition $\left|6 \mathrm{P}_{3 / 2}, F^{\prime}=5\right\rangle \rightarrow\left|56 \mathrm{D}_{5 / 2}\right\rangle$. A 3.99-GHz microwave field couples the Rydberg transition $\left|56 \mathrm{D}_{5 / 2}\right\rangle \rightarrow\left|57 \mathrm{P}_{3 / 2}\right\rangle$, yielding microwave dressed Rydberg electromagnetically inducted transparency (EIT)-Autler-Townes (AT) spectra. (b) Schematic of the experiment. The probe and coupling lasers are overlapped in a counter propagating geometry inside an atomic probe (caesium vapor cell). The probe beam is passed through a dichroic mirror (DM) and is detected with a photodiode (PD). The distance from the center of the atom probe to the guide rail is $d_{0}=0.3 \mathrm{~m}$. The horn can be translated along the rail. $d$ is the distance between the horn and the center of the rail. Not to scale.

A standard gain horn with center frequency of $\sim 4.0 \mathrm{GHz}$ is placed on a commercial one-dimensional guide rail. The rail is placed parallel to the laser beams. The cell center is located along the perpendicular bisector of the rail with the distance $d_{0}=0.3 \mathrm{~m}$, as shown in Figure $1 \mathrm{~b}$. The vertical center of the horn is positioned in the optical plane. We change the horn position $d$ by moving it along the rail, and detect the microwave induced EIT-AT splitting. The strength of the splitting allows us to obtain the electric field value.

A 3.99 GHz microwave electric field, produced with an analog signal generator (Agilent N5183B), is emitted by the horn. This field is incident upon the cell, transverse to the counter-propagating probe and coupling beams. The microwave field couples nearby Rydberg transition $\left|56 D_{5 / 2}\right\rangle \rightarrow\left|57 P_{3 / 2}\right\rangle$ and splits the EIT peak. For investigating the near field feature of the horn, we fix the output of the microwave source at $13 \mathrm{dBm}$. At this power, EIT splitting is observable at all horn positions, $d \in[-0.4 \mathrm{~m}, 0.4 \mathrm{~m}]$.

\section{Results and Discussion}

In Figure 2b,c, we present microwave dressed AT spectra with the $3.99 \mathrm{GHz}$ microwave source power fixed at $13 \mathrm{dBm}$. The horn is placed at $d=+0.24 \mathrm{~m}$ and $-0.09 \mathrm{~m}$, respectively. The microwave dressed AT splitting, $\gamma_{A T}$, is defined as the peak-to-peak distance of the Rydberg EIT-AT line pair, which is extracted using the multipeak Lorentz fittings, denoted as solid lines of Figure 2b,c. The fittings to the EIT-AT spectra yield $\gamma_{A T}=2 \pi \times 55.50 \mathrm{MHz}$ in Figure $2 \mathrm{~b}$ and $2 \pi \times 103.52 \mathrm{MHz}$ in Figure 2c. As AT splitting is proportional to the the Rabi frequency [9], the microwave dressed AT splitting is 
proportional to the microwave coupling, $2 \pi \gamma_{A T}=\Omega_{M W}$. We can then obtain the microwave electric field amplitude via the equation,

$$
E=\frac{\hbar}{\mu_{M W}} \Omega_{\mathrm{MW}}=2 \pi \frac{\hbar}{\mu_{M W}} \gamma_{A T} .
$$

The AT splittings of Figure $2 b, c$ yield microwave electric fields of 2.1 and $3.9 \mathrm{~V} / \mathrm{cm}$, respectively. It is noted that in addition to the peaks indicative of microwave dressed AT splitting, there is an additional peak at zero-detuning. This peak is attributed to the magnetic sub-levels of $56 D_{5 / 2}$ state that are not coupled to the $57 \mathrm{P}_{3 / 2}$ state in the linear polarized microwave field or the component of the microwave field that is perpendicular to the laser polarization. Our experiment is the latter case that indicates that the microwave field has a slight elliptical polarization. The magnitude of this component increases at large $d$, causing the central peak to reduce in size. For details see [33].

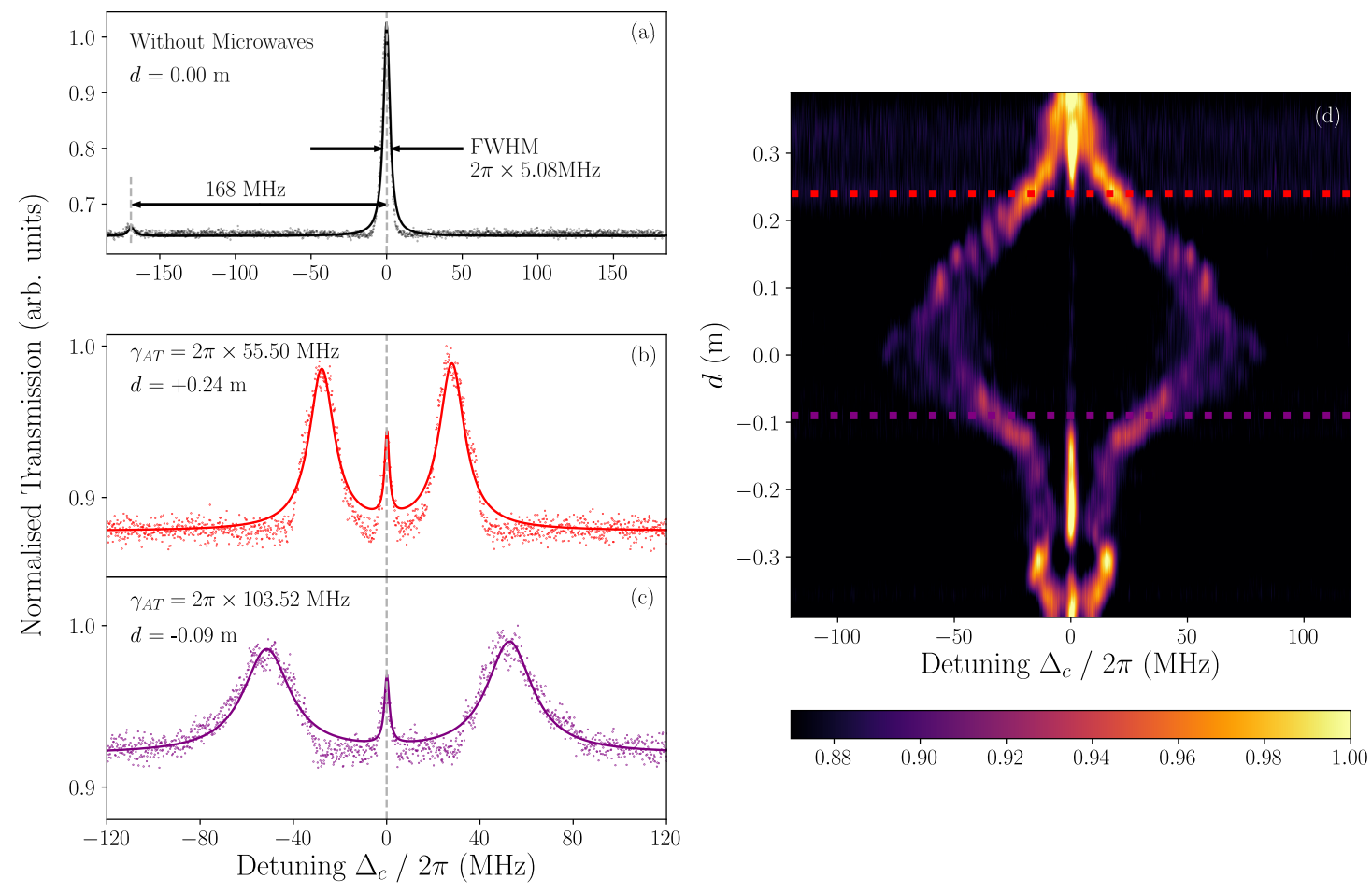

Figure 2. (Color online) (a) $\Omega_{M W}=0$ Rydberg EIT spectrum. The main peak at zero detuning corresponds to the $\left|6 \mathrm{~S}_{1 / 2}, F=4\right\rangle \rightarrow\left|6 \mathrm{P}_{3 / 2}, F^{\prime}=5\right\rangle \rightarrow\left|56 \mathrm{D}_{5 / 2}\right\rangle$ cascade EIT spectrum. The small peak at $-168 \mathrm{MHz}$ results from the intermediate-state hyperfine level $\left|6 \mathrm{P}_{3 / 2}, F^{\prime}=4\right\rangle$, and is used to calibrate the coupling laser frequency. $(\mathbf{b}, \mathbf{c})$ Microwave dressed Rydberg EIT-AT spectra as a function of the coupling laser detuning, $\Delta_{\mathrm{c}}$, for the horn position at $d=+0.24 \mathrm{~m}(\mathbf{b})$ and $d=-0.09 \mathrm{~m}(\mathbf{c})$, respective AT splittings are $\gamma_{A T} / 2 \pi=55.50 \mathrm{MHz}$ and $103.52 \mathrm{MHz}$. AT splitting, $\gamma_{A T}$, is defined as the peak-to-peak separation of the microwave dressed AT line pair, which are extracted using the multi-peak Lorentz fittings (solid lines). The frequency of the microwave field is $3.99 \mathrm{GHz}$ driving the Rydberg transition $\left|56 \mathrm{D}_{5 / 2}\right\rangle \rightarrow\left|57 \mathrm{P}_{3 / 2}\right\rangle$. The small peak at zero detuning originates from the component of the microwave field that is perpendicular to the laser polarization. (d) Measurements of microwave dressed EIT-AT spectra for the range of $|d| \leq 0.39 \mathrm{~m}$. Microwave dressed AT line strength is displayed using a linear color scale. Two horizontal dashed lines are shown the case of (b) (red) and (c) (purple), respectively.

To investigate the distribution of the microwave field emitted by the horn, we perform a series of measurements at horn positions $d$ between $d_{\max }= \pm 0.4 \mathrm{~m}$ with a step size of $0.03 \mathrm{~m}$. The microwave dressed AT spectrum is displayed with a linear color scale in Figure 2d. We find that (i) the microwave dressed AT splitting is largest at $d=0$. Here, atoms experience the largest microwave field. (ii) Closer 
inspection of the spectrum at $d=0$ in Figure $2 \mathrm{~d}$ reveals that the peak at 0-detuning (associated with polarization impurities) is small relative to Figure $2 \mathrm{~b}, \mathrm{c}$, where the horn is at $d=+0.24 \mathrm{~m}$ and $-0.09 \mathrm{~m}$. This demonstrates that the Rydberg atoms experience a microwave field with a relatively pure linear polarization parallel to the polarization of laser beams when the horn is at $d=0$, and we clearly observe the two peak EIT-AT spectrum [22]. Meanwhile the AT spectrum at $|d| \approx 0$ experiences a strong ac Stark effect, and causes additional splitting of the EIT feature which can be seen on the figure at small $d$. The Lorentzian nature of the AT split peaks is also degraded by inhomogeneity of the microwave field across the cell. Rydberg atoms within the cell experience slightly different microwave fields, leading to a slightly different AT splittings [34]. We use multi-peak Lorentz fittings to fit all peaks, the averaged AT splitting (electric field) is considered as the microwave field at near $d=0$. (iii) The microwave dressed AT splitting decreases with $d$, whereas the strength of 0-detuning peak increases with $d$, as the spectrum converges on the zero detuning case. The quality of the polarization also decreases at large $d$, increasing this effect.

In Figure 3a, we show measurements of microwave electric-field, $\lg \left[E / E_{0}\right] \simeq \lg \left[\gamma_{A T} / \gamma_{A T}^{d=0}\right]$ with $E_{0}\left(\gamma_{A T}^{d=0}\right)$ taken as the value the electric field (AT splitting) experienced by the atoms when the horn is positioned at $d=0$. The Stark effect is largest at $d=0$ and decreases with $|d|$. For comparison, in Figure 3a we also plot measurements taken with a dipole metal probe and analyzed with a vector network analyzer (VNA). The metal probe was placed at the same position as the atomic cell. Measurements with the atomic probe are consistent with the dipole metal probe over the measurement range.

To compare the measurements, we calculate the near field distribution using an EMSS based on electromagnetic theory, which is solved with Maxwell's equations and proper boundary conditions $[35,36]$. The solid line of Figure 3 a displays this simulation of the near field. The figure shows that both the atom- and metal-probe measurements are in agreement with the simulations. The atomic probe outperforms the metal probe; the measurements obtained therewith more accurately reproduce the simulated field. This is probably because the metal probe itself reflects the microwave field that perturbs the field under measurement. A grey box on Figure 3a highlights the data taken with our atomic probe that are in agreement with the simulations. Outside of this grey box, the field value is too small to be measured accurately. For $d \gtrsim \pm 0.25 \mathrm{~m}$, the side lobes of the measured field do not vanish as rapidly as the simulations predict. Here, the measured field diverges from simulations. When $d>>0$, the electric field experienced by the Rydberg atoms is weak, susceptible to interference from reflected microwaves and the environment. We display residuals $R$ between experiment and simulation for the gray region in the lower panel of Figure 3a. Corresponding root mean square (RMS) values of residuals are respectively $1.1 \mathrm{~dB}$ and $2.7 \mathrm{~dB}$ for atom- and metal-probe measurements, the atom probe is, therefore a $1.6 \mathrm{~dB}$ improvement over measurements taken with the metal probe. Further in Figure $3 \mathrm{~b}$, we present the radiation pattern of the horn used here (electric field, $E / E_{0}$, as a function of $\left.\theta\left(\arctan \left[d / d_{0}\right]\right)\right)$. The dot-dashed lines display the half power beam width (HPBW). The HPBW is defined as the angle between the two directions where the electric field decreases to $E_{0} / \sqrt{2}$. Measured HPBW is $(39.4 \pm 3.8)^{\circ}$ for atom probe, again providing a more accurate measurement $\left(\mathrm{HPBW}_{E M S S}=33.6^{\circ}\right)$ than the metal probe $(9.2 \pm 2.2)^{\circ}$.

We then analyze the symmetry of the near field of the microwave horn. Figure 4 displays the measurements of the normalized microwave electric field, $\lg \left[E / E_{0}\right]$, as a function of $|d|$ so that the symmetry of the near field of the horn can be addressed. We also plot the simulated electric field versus $d$, which is the same for the sides, see black line in Figure 4. Short-dashed lines show the fittings to the experimental data using the equation $y=a+b /\left(d_{0}^{2}+d^{2}\right)$, with fitting parameters $a$ and $b$. Parameters $a_{\text {right }}=-26.14 \pm 1.10, a_{\text {left }}=-26.12 \pm 1.88$ and $b_{\text {right }}=2.42 \pm 0.13, b_{\text {left }}=2.32 \pm 0.23$ are obtained. The minor difference between these parameters for both sides demonstrates some asymmetry of the near field distribution. From Figures 3 and 4, it is seen that the simulated microwave electric field shows a symmetrical distribution of the near field. Measurements at larger $d$ demonstrate 
some asymmetry. This is attributed to reflections of the microwave field from optics, and noise from the environment.
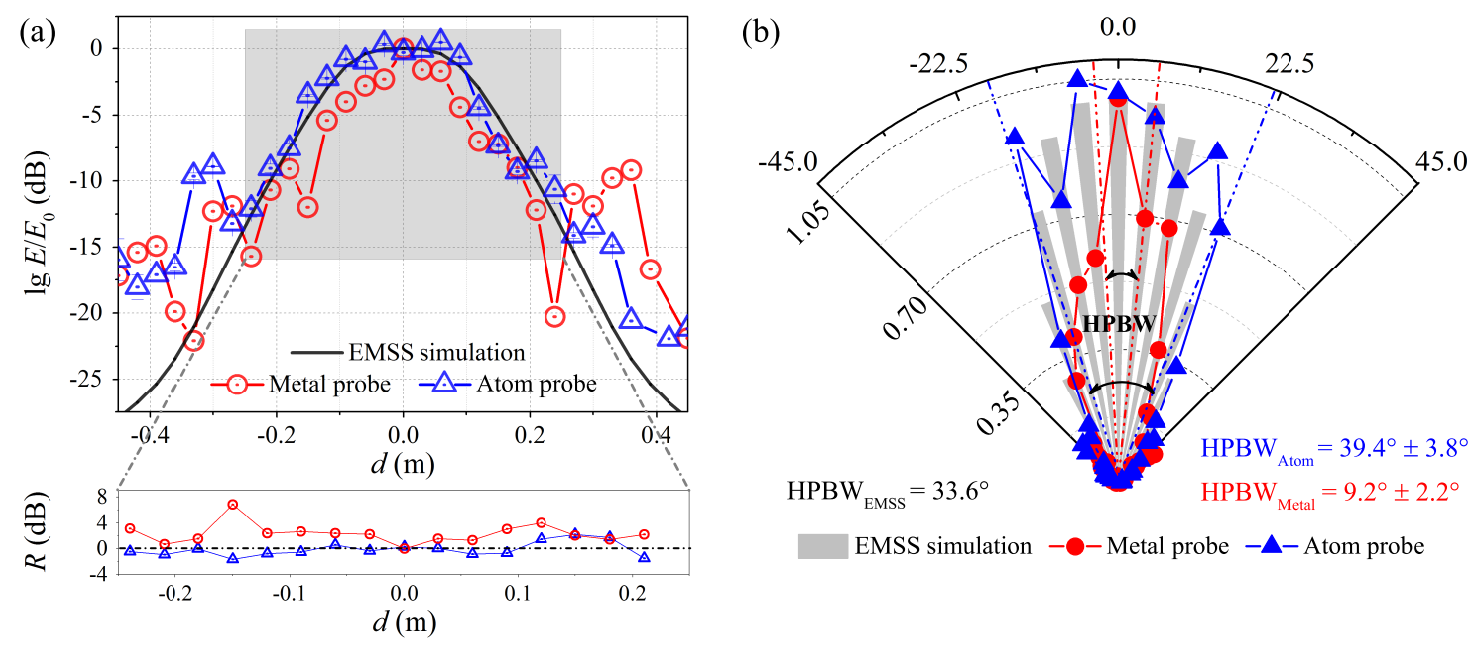

Figure 3. (Color online) (a) Electric field amplitude $\lg \left[E / E_{0}\right]\left(E_{0}\right.$, microwave field at $\left.d=0\right)$ measured using an atomic probe (blue triangles) and a dipole metal probe (red circles). The error bars for atomic measurements display a standard error of two measurements. The solid line displays the simulation with the electromagnetic simulation software. The gray shadowed area corresponds to $d= \pm 0.25 \mathrm{~m}$. The lower panel shows the residuals $R$ between experiment and theory within this grey area. The root mean square (RMS) values of the residuals are $1.1 \mathrm{~dB}$ and $2.7 \mathrm{~dB}$ for the atomic and metal probe, respectively. (b) Comparison of the electric field, $\left[E / E_{0}\right]$ between measurements and electromagnetic simulated software (EMSS) simulation at near field. The major lobe of the emission is observed with measured half power beam width (HPBW) $(39.4 \pm 3.8)^{\circ}$ for the atom probe and $(9.2 \pm 2.2)^{\circ}$ for the metal probe.

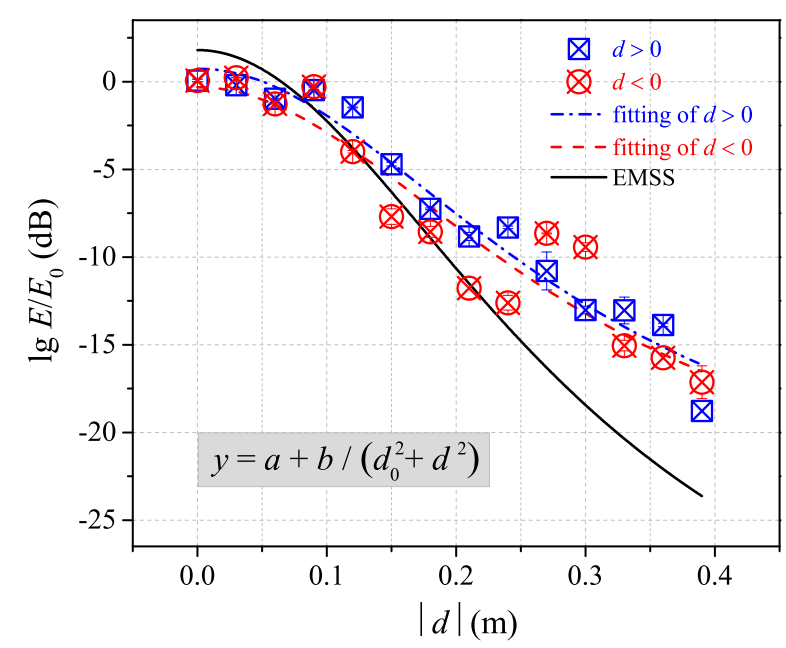

Figure 4. (Color online) The measured electric field, $\lg \left[E / E_{0}\right]$, as a function of distance from the center of the guide rail to the horn, $d$, for both directions $(d>0$ and $d<0)$. The error bars for atomic measurements display a standard error of two measurements. Short-dashed lines are the fittings to the experimental data using an equation $y=a+b /\left(d_{0}^{2}+d^{2}\right)$ with parameters $a$ and $b$. The fitting parameters are $a_{\text {right }}=-26.14 \pm 1.10, b_{\text {right }}=2.42 \pm 0.13$ and $a_{\text {left }}=-26.12 \pm 1.88, b_{\text {left }}=2.32 \pm 0.23$, respectively. The minor difference in the fitting parameters for the data describing the right and left sides of the microwave distribution is symptomatic of a small asymmetry of the near field. The thin line denotes the simulation based on EMSS. 
The microwave dressed Rydberg EIT-AT spectrum provides an optical readout of the microwave electric field that is employed to measure the near-field distribution of the horn. The microwave dressed AT spectrum reveals both the microwave field and polarization purity of the microwave horn. From Figure 3, we have well characterized the amplitude distribution and radiation pattern at the near field of a microwave horn using AT splitting of sensitive Rydberg states. From Figure 2d, we can also determine the purity of the microwave polarization by observing the presence and size of the central peak. We realize a real-time measurement and monitoring of the microwave horn field able to produce measurements of microwave fields in just seconds. Using a smaller cell would improve the spatial resolution of the measurement and so would improve the accuracy of near field measurements of more complicated fields. This technique can easily be extended to two and three dimensional field measurements, paving the way for the investigation of microwave field distributions.

\section{Conclusions}

Using a room temperature caesium vapor cell as an atomic probe, we have measured the near field of a microwave horn emitting microwaves at $3.99 \mathrm{GHz}$ by analyzing microwave dressed EIT-AT spectra. The caesium three-level system involving Rydberg $56 \mathrm{D}_{5 / 2}$ is employed to provide an optical measurement of the AT splitting of a Rydberg EIT feature, due to the coupling between the $\left|56 \mathrm{D}_{5 / 2}\right\rangle \rightarrow$ $\left|57 \mathrm{P}_{3 / 2}\right\rangle$ transition caused by the microwave field. This Rydberg atom sensor provides information on both the amplitude and polarization of the microwave field. Furthermore, we measure the electric field distribution of the near field by changing the position of the microwave horn. A set of measurements of the same field is obtained using a standard dipole metal probe. Both sets of measurements are compared to EMSS simulations. We find that the measurement of the atom probe is a better match to the EMSS simulations than measurements taken with the dipole metal probe. Residuals of the atomic probe data are $1.6 \mathrm{~dB}$ smaller compared to the metal probe. This Rydberg-atom probe has applications in microwave electric field and polarization measurements, and allows accurate and precise investigation of the distribution of complex microwave fields.

Author Contributions: Conceptualization, J.Z., Y.J. and J.B.; investigation, J.Z., Y.J. and J.B.; software, Y.J., J.F. and N.L.R.S.; validation, J.B., L.H. and J.F.; formal analysis, J.B., J.F. and L.H.; Writing-original draft, J.B.; writing-review and editing, J.Z.; project administration, J.Z. and Y.J.; funding acquisition, J.Z. and Y.J.

Funding: The work was supported by the National Key R\&D Program of China (Grant No. 2017YFA0304203), the State Key Program of National Natural Science of China (Grant No. 11434007 and 61835007), the National Natural Science Foundation of China (Grants No. 61675123, No. 61775124 and 11804202), and Changiiang Scholars and Innovative Research Team in University of Ministry of Education of China (Grant No. IRT_17R70).

Acknowledgments: We thank Zhenghui Xue and Qingyun Zeng for helpful discussions on EMSS.

Conflicts of Interest: The authors declare no conflict of interest.

\section{References}

1. Kanda, M.; Driver, L.D. An Isotropic Electric-Field Probe with Tapered Resistive Dipoles for Broad-Band Use, $100 \mathrm{kHz}$ to $18 \mathrm{GHz}$. IEEE Trans. Microw. Theory Tech. 1987, 35, 124-130. [CrossRef]

2. Holloway, C.L.; Gordon, J.A.; Jefferts, S.; Schwarzkopf, A.; Anderson, D.A.; Miller, S.A.; Thaicharoen, N.; Raithel, G. Broadband Rydberg Atom-Based Electric-Field Probe for SI-Traceable, Self-Calibrated Measurements. IEEE Trans. Antenn. Propag. 2014, 62, 6169-6182. [CrossRef]

3. Kanda, M. Standard Probes for Electromagnetic Field Measurements. IEEE Trans. Antenn. Propag. 1993, 41, 1349-1364. [CrossRef]

4. Hall, J.L. Nobel Lecture: Defining and measuring optical frequencies. Rev. Mod. Phys. 2006, 78, 1279. [CrossRef]

5. Bloom, B.J.; Nicholson, T.L.; Williams, J.R.; Campbell, S.L.; Bishof, M.; Zhang, X.; Zhang, W.; Bromley, S.L.; Ye, J. An optical lattice clock with accuracy and stability at the $10^{-18}$ level. Nature 2014, 506, 71. [CrossRef]

6. Savukov, I.M.; Seltzer, S.J.; Romalis, M.V.; Sauer, K.L. Tunable Atomic Magnetometer for Detection of Radio-Frequency Magnetic Fields. Phys. Rev. Lett. 2005, 95, 063004. [CrossRef] 
7. Patton, B.; Versolato, O.O.; Hovde, D.C.; Corsini, E.; Higbie, J.M.; Budker, D. A remotely interrogated all-optical ${ }^{87} \mathrm{Rb}$ magnetometer. Appl. Phys. Lett. 2012, 101, 083502. [CrossRef]

8. Gallagher, T.F. Rydberg Atoms; Cambridge University Press: New York, NY, USA, 1994.

9. Autler, S.H.; Townes, C.H. Stark Effect in Rapidly Varying Fields. Phys. Rev. 1955, 100, 703. [CrossRef]

10. Mohapatra, A.K.; Jackson, T.R.; Adams, C.S. Coherent Optical Detection of Highly Excited Rydberg States Using Electromagnetically Induced Transparency. Phys. Rev. Lett. 2007, 98, 113003. [CrossRef]

11. Grimmel, J.; Mack, M.; Karlewski, F.; Jessen, F.; Reinschmidt, M.; Sándor, N.; Fortágh, J. Measurement and numerical calculation of Rubidium Rydberg Stark spectra. New J. Phys. 2015, 17, 053005. [CrossRef]

12. Mack, M.; Karlewski, F.; Hattermann, H.; Höckh, S.; Jessen, F.; Cano, D.; Fortágh, J. Measurement of absolute transition frequencies of ${ }^{87} \mathrm{Rb}$ to $n S$ and $n D$ Rydberg states by means of electromagnetically induced transparency. Phys. Rev. A 2011, 83, 052515. [CrossRef]

13. Fan, H.Q.; Kumar, S.; Sedlacek, J.; Kübler, H.; Karimkashi, S.; Shaffer, J.P. Atom based RF electric field sensing. J. Phys. B At. Mol. Opt. Phys. 2015, 48, 202001. [CrossRef]

14. Song, Z.F.; Liu, H.P.; Liu, X.C.; Zhang, W.F.; Zou, H.Y.; Zhang, J.; Qu, J.F. Rydberg-atom-based digital communication using a continuously tunable radio-frequency carrier. Opt. Express 2019, 27, 8848-8857. [CrossRef] [PubMed]

15. Jiao, Y.C.; Han, X.X.; Fan, J.B.; Raithel, G.; Zhao, J.M.; Jia, S.T. Atom-based quantum receiver for amplitude- and frequency-modulated baseband signals in high-frequency radio communication. arXiv 2018, arXiv:1804.07044v1.

16. Anderson, D.A.; Paradis, E.; Raithel, G.; Sapiro, R.E.; Simons, M.T.; Holloway, C.L. High-resolution near-field imaging and far-field antenna measurements with atomic sensors. In Proceedings of the International Symposium on Electromagnetic Compatibility (EMC Europe), Amsterdam, The Netherlands, 27-30 August 2018; p. 391.

17. Cox, K.C.; Meyer, D.H.; Fatemi, F.K.; Kunz, P.D. Quantum-Limited Atomic Receiver in the Electrically Small Regime. Phys. Rev. Lett. 2018, 121, 110502. [CrossRef]

18. Shylla, D.; Nyakang'o, E.O.; Pandey, K. Highly sensitive atomic based MW interferometry. Sci. Rep. 2018, 8, 8692. [CrossRef]

19. Jiao, Y.C.; Hao, L.P.; Han, X.X.; Bai, S.Y.; Raithel, G.; Zhao, J.M.; Jia, S.T. Atom-Based Radio-Frequency Field Calibration and Polarization Measurement Using Cesium $n D_{J}$ Floquet States. Phys. Rev. Appl. 2017, 8, 014028. [CrossRef]

20. Kumar, S.; Fan, H.Q.; Kübler, H.; Jahangiri, A.J.; Shaffer, J.P. Rydberg-atom based radio-frequency electrometry using frequency modulation spectroscopy in room temperature vapor cells. Opt. Express 2017, 25, 8625. [CrossRef]

21. Kumar, S.; Fan, H.Q.; Kübler, H.; Sheng, J.T.; Shaffer, J.P. Atom-Based Sensing of Weak Radio Frequency Electric Fields Using Homodyne Readout. Sci. Rep. 2017, 7, 42981. [CrossRef]

22. Anderson, D.A.; Raithel, G. Continuous-frequency measurements of high-intensity microwave electric fields with atomic vapor cells. Appl. Phys. Lett. 2017, 111, 053504. [CrossRef]

23. Veit, C.; Epple, G.; Kübler, H.; Euser, T.G.; Russell, P.S.J.; Löw, R. RF-dressed Rydberg atoms in hollow-core fibres. J. Phys. B At. Mol. Opt. Phys. 2016, 49, 134005. [CrossRef]

24. Jiao, Y.C.; Han, X.X.; Yang, Z.W.; Li, J.K.; Raithel, G.; Zhao, J.M.; Jia, S.T. Spectroscopy of cesium Rydberg atoms in strong radio-frequency fields. Phys. Rev. A 2016, 94, 023832. [CrossRef]

25. Fan, H.Q.; Kumar, S.; Sheng, J.T.; Shaffer, J.P. Effect of Vapor-Cell Geometry on Rydberg-Atom-Based Measurements of Radio-Frequency Electric Fields. Phys. Rev. Appl. 2015, 4, 044015. [CrossRef]

26. Fan, H.Q.; Kumar, S.; Daschner, R.; Kübler, H.; Shaffer, J.P. Subwavelength microwave electric-field imaging using Rydberg atoms inside atomic vapor cells. Opt. Lett. 2014, 39, 3030. [CrossRef]

27. Holloway, C.L.; Gordon, J.A.; Schwarzkopf, A.; Anderson, D.A.; Miller, S.A.; Thaicharoen, N.; Raithel, G. Sub-wavelength imaging and field mapping via electromagnetically induced transparency and Autler-Townes splitting in Rydberg atoms. Appl. Phys. Lett. 2014, 104, 244102. [CrossRef]

28. Zhang, H.; Zhang, L.J.; Wang, L.M.; Bao, S.X.; Zhao, J.M.; Jia, S.T. Autler-Townes spectroscopy with interaction-induced dephasing. Phys. Rev. A 2014, 90, 043849. [CrossRef]

29. Sedlacek, J.A.; Schwettmann, A.; Kübler, H.; Löw, R.; Pfau, T.; Shaffer, J.P. Microwave electrometry with Rydberg atoms in a vapour cell using bright atomic resonances. Nat. Phys. 2012, 8, 819. [CrossRef] 
30. Downes, L.A.; MacKellar, A.R.; Whiting, D.J.; Bourgenot, C.; Adams, C.S.; Weatherill, K.J. Ultra-high-speed Terahertz Imaging using Atomic Vapour. arXiv 2019, arXiv:1903.01308v2.

31. Wade, C.G.; Šibalić, N.; Melo, N.R.d.; Kondo, J.M.; Adams, C.S.; Weatherill, K.J. Real-time near-field terahertz imaging with atomic optical fluorescence. Nat. Photonics 2016, 11, 40. [CrossRef]

32. Pearman, C.P.; Adams, C.S.; Cox, S.G.; Griffin, P.F.; Smith, D.A.; Hughes, I.G. Polarization spectroscopy of a closed atomic transition: Applications to laser frequency locking. J. Phys. B At. Mol. Opt. Phys. 2002, 35, 5141. [CrossRef]

33. Sedlacek, J.A.; Schwettmann, A.; Kübler, H.; Shaffer, J.P. Atom-Based Vector Microwave Electrometry Using Rubidium Rydberg Atoms in a Vapor Cell. Phys. Rev. Lett. 2013, 111, 063001. [CrossRef] [PubMed]

34. Fan, J.B.; Jiao, Y.C.; Hao, L.P.; Xue, Y.M.; Zhao, J.M.; Jia, S.T. Microwave electromagnetically induced transparency and Aulter-Townes spectrum of cesium Rydberg atom. Acta Phys. Sin. 2018, 9, 093201.

35. Hansen, T.B.; Yaghjian, A.D. Planar near-field scanning in the time domain .1. Formulation. IEEE Trans. Antenn. Propag. 1994, 42, 1280. [CrossRef]

36. Hansen, T.B.; Yaghjian, A.D. Planar near-field scanning in the time domain .2. Sampling theorems and computation schemes. IEEE Trans. Antenn. Propag. 1994, 42, 1292. [CrossRef]

(C) 2019 by the authors. Licensee MDPI, Basel, Switzerland. This article is an open access article distributed under the terms and conditions of the Creative Commons Attribution (CC BY) license (http:/ / creativecommons.org/licenses/by/4.0/). 